Research, Society and Development, v. 10, n. 8, e60010817687, 2021

(CC BY 4.0) | ISSN 2525-3409 | DOI: http://dx.doi.org/10.33448/rsd-v10i8.17687

\title{
Cartas terapêuticas: Tecnologia estratégica de comunicação e seus desdobramentos
}

\section{terapêuticos}

\author{
Therapeutic letters: Strategic communication technology and its therapeutic consequences \\ Letras terapéuticas: Tecnología de la comunicación estratégica y sus consecuencias terapêuticas
}

Recebido: 29/06/2021 | Revisado: 05/07/2021 | Aceito: 08/07/2021 | Publicado: 18/07/2021

\author{
Juliana de Souza Ferreira \\ ORCID: https://orcid.org/0000-0002-3064-542X \\ Universidade Federal do Estado do Rio de Janeiro, Brasil \\ E-mail: julianaferreira.ufrj@gmail.com \\ Larissa Bollorini Cypriano \\ ORCID: https://orcid.org/0000-0002-9218-7209 \\ Universidade Federal do Estado do Rio de Janeiro, Brasil \\ E-mail: larissabollorini@hotmail.com \\ Nayara Araujo de Souza Vasconcellos \\ ORCID: https://orcid.org/0000-0001-9902-8959 \\ Universidade Federal do Estado do Rio de Janeiro, Brasil \\ E-mail: araujos.nayara@gmail.com \\ Gabriela Malinosky Sepulvida \\ ORCID: https://orcid.org/0000-0002-0427-1719 \\ Universidade Federal do Estado do Rio de Janeiro, Brasil \\ E-mail: gabimalinosky@gmail.com \\ João Victor Lima da Silva \\ ORCID: https://orcid.org/0000-0002-5561-0303 \\ Universidade Federal Fluminense, Brasil \\ E-mail: lima21@outlook.com \\ Adriana da Costa Coelho \\ ORCID: https://orcid.org/0000-0003-2047-9592 \\ Universidade Federal do Estado do Rio de Janeiro, Brasil \\ E-mail: dricoelho10@hotmail.com \\ Eliza Cristina Macedo \\ ORCID: https://orcid.org/0000-0001-8824-9107 \\ Universidade Federal do Estado do Rio de Janeiro, Brasil \\ E-mail:_macedo.unirio@gmail.com \\ Renata Almeida Martins \\ ORCID: https://orcid.org/0000-0001-5999-3156 \\ Universidade Federal do Rio de Janeiro, Brasil \\ E-mail: renata_martins21@hotmail.com \\ Juliana Roza Dias \\ ORCID: https://orcid.org/0000-0002-6615-3603 \\ Universidade do Estado do Rio de Janeiro, Brasil \\ E-mail: jullyroza2003@yahoo.com.br
}

\begin{abstract}
Resumo
Objetivo: identificar na literatura científica o uso de cartas terapêuticas como tecnologia facilitadora da comunicação entre a equipe de enfermagem e pacientes. Metodologia: trata-se de uma pesquisa de abordagem qualitativa do tipo revisão integrativa da literatura, realizada nos meses de dezembro de 2020 e janeiro de 2021. Resultados: após a busca nas bases de dados Cinahl, Medline e Scopus, foram selecionados 9 artigos para compor a amostra final desta revisão. Conclusão: é inovadora e diferencial a aplicabilidade das cartas terapêuticas para relações interpessoais, o que pode evidenciar um cuidado mais humanizado por parte da equipe de enfermagem, motivando o paciente a entender que a equipe está junto a ele em todo o processo de recuperação e tratamento, estabelecendo um vínculo de confiança, referência e contrarreferência na assistência. É escassa a produção científica sobre o assunto supracitado e portanto, diminuído está o embasamento científico para esta obra, por esse motivo é necessário o incentivo a esses assuntos referenciados ao cunho holístico do ser humano.
\end{abstract}

Palavras-chave: Cartas terapêuticas; Saúde; Saúde mental. 


\begin{abstract}
Objective: to identify scientific production or the use of therapeutic letters as a technology that facilitates communication between the nursing team and the patient. Methodology: this is a qualitative approach research of the integrative literature review type, carried out between December 2020 and January 2021. Results: search in Cinahl, Medline and Scopus databases, selected by 9 articles to Compose the final sample of this review. Conclusion: it is innovative and differential regarding the applicability of therapeutic letters for interpersonal relationships, or that it can demonstrate a more humanized care by the nursing team, motivating the patient to understand that the team is close to the entire recovery and treatment process, establishing a bond of trust, referral and counter-referral for assistance. There is little scientific production on the subject in question and, therefore, there is little scientific basis for this work, so it is necessary to either encourage these subjects referenced or as holistic human beings.
\end{abstract}

Keywords: Therapeutic letters; Health; Mental health.

\title{
Resumen
}

Objetivo: identificar en la literatura científica el uso de letras terapéuticas como tecnología que facilita la comunicación entre el equipo de enfermería y los pacientes. Metodología: se trata de una investigación de abordaje cualitativo del tipo revisión integradora de la literatura, realizada en diciembre de 2020 y enero de 2021 . Resultados: luego de buscar en las bases de datos Cinahl, Medline y Scopus, se seleccionaron 9 artículos para componer la muestra final de esta revisión. Conclusión: la aplicabilidad de las cartas terapéuticas a las relaciones interpersonales es innovadora y diferencial, lo que puede mostrar un cuidado más humanizado por parte del equipo de enfermería, motivando al paciente a entender que el equipo está con él durante todo el proceso de recuperación y tratamiento, estableciendo un vínculo de confianza, derivación y contrarreferencia en la atención. La producción científica sobre el tema mencionado es escasa y, por tanto, la base científica para este trabajo se ve mermada, por ello es necesario incentivar estos temas relacionados con la naturaleza holística del ser humano.

Palabras clave: Letras terapêuticas; Salud; Salud mental.

\section{Introdução}

A doença é um marco referencial na vida de uma pessoa em um processo de construção e desconstrução de experiências. É um momento de extrema delicadeza para todos envolvidos nesse processo: profissionais, pacientes e família.

A família, para Fernandes et al., (2015), é um importante alicerce externo na recuperação do paciente internado. No entanto, em situações extremamente novas, como é o caso da pandemia da Covid-19, medidas de segurança e de contenção da transmissão da virulência no âmbito hospitalar devem ser tomadas, tais como a suspensão de visitas, que provocam maior isolamento social dos enfermos, requerendo um maior apoio da equipe de saúde para o enfrentamento da doença neste contexto.

A construção de uma estratégia de comunicação entre a equipe e o paciente é imprescindível para auxiliá-lo na sua recuperação e subsidiar cuidado integral e humanizado. Neste cenário, a carta terapêutica entra como tecnologia facilitadora na troca de experiências entre os profissionais da equipe de enfermagem e os enfermos. Além de ser meio de promover mudanças, envolvendo esforços particulares, concepções motivacionais e resgate da comunicação face a face (MAIO, 2020).

As cartas terapêuticas se distinguem das cartas sociais, segundo Oliver et al., (2007), pelo cerne do cenário, circunstâncias, propósitos e repercussões provocadas no paciente e na equipe. As mensagens são mais literárias do que a investigação clínica do paciente, revelando mais episódios e perspectiva do mesmo.

Alguns estudos de Hunt, Schochet \& King (2005), apontam que o ato de escrever cartas tidas como terapêuticas foi iniciado por Freud, com o principal intuito de responder sobre questões transferenciais. Sigmund Freud (1893-1895), em suas obras cita o uso dessa estratégia como recurso para a prática clínica, sendo entendida ao mesmo tempo, como forma de documentação e de intervenção terapêutica. O ser humano é essencialmente um ser relacional, e para a ocorrência do processo amistoso de uma relação interpessoal é exigida uma troca psíquica que favoreça este processo. Na psicanálise, o médico traz o conceito da transferência como sendo o deslocamento do sentido atribuído à pessoa do passado para pessoas do nosso presente, sendo executada pelo inconsciente. Para ele esse fenômeno é fundamental para o processo de cura. Cita-se este conceito para 
Research, Society and Development, v. 10, n. 8, e60010817687, 2021

(CC BY 4.0) | ISSN 2525-3409 | DOI: http://dx.doi.org/10.33448/rsd-v10i8.17687

trazer a partir dele, a importância da construção de vínculos positivos entre enfermeiro/médico - paciente. Sentimentos como sensibilidade, humanismo e amor ao próximo são fundamentais para o sucesso de um tratamento.

Segundo Paiva \& Rasera (2012) nesse contexto teórico, é possível visualizar a contribuição ética das cartas terapêuticas à medida que se entende que a verdade não está no profissional tampouco no que ele escreve.

Existem poucos estudos sobre cartas terapêuticas, mas Vidgen \& Williams (2001) reforçam que há relevância na temática. Assim, os subsídios do uso das cartas terapêuticas são as peculiaridades pertinentes à escrita, suas finalidades, bem como os vários modelos e a utilização de cartas. Ressalta-se que as cartas, como um instrumento de melhoria da relação interpessoal, é uma tecnologia facilitadora entre a equipe de enfermagem e pacientes internados. Representando uma estratégia para auxiliá-lo na sua recuperação, configurando-se assim como uma importante intervenção terapêutica.

Durante o novo cenário pandêmico, pode-se observar a tentativa de prevenção da saúde física das pessoas, dessa forma foram adotadas medidas, a fim de diminuir o fluxo de pessoas transitando, com a suspensão de procedimentos cirúrgicos eletivos e remanejamento de atividades não essenciais para ambiente extra-hospitalar. O objetivo deste isolamento foi achatar a curva de transmissão, possibilitando o atendimento hospitalar das pessoas de forma plena, disponibilizando recursos materiais e humanos necessários para o cuidar, porém estas mudanças visam apenas a saúde física e a saúde mental é negligenciada ou subestimada (OrnelL et al., 2020).

Por esse motivo é necessário entender além das repercussões físicas provocadas pelo COVID-19, como síndrome respiratória aguda grave, os impactos vão além e atingem a saúde mental dos indivíduos. Diferentes fatores podem estar envolvidos, como medo da contaminação, estresse financeiro, efeitos adversos ao tratamento e isolamento social. Desta forma, é necessário e imprescindível a atenção e o acompanhamento dos desdobramentos patológicos na saúde mental do homem, tratando-o de forma holística e longitudinal, de acordo com as demandas que este apresente (Raony et al., 2020; Schmidt et al., 2020).

A grande contribuição do estudo é explicitada fortemente pelo princípio da equidade, especialmente pela natureza subjetiva, a qual, cada sujeito interpreta a fase vivenciada. A implementação de estratégias terapêuticas no cenário atual propicia um cuidado holístico. Sendo assim, as cartas podem ser utilizadas como ferramenta na assistência pela equipe de enfermagem.

Frente ao exposto, este estudo tem como objetivo identificar na literatura científica o uso de cartas terapêuticas como tecnologia facilitadora da comunicação entre a equipe de enfermagem e pacientes.

\section{Metodologia}

Trata-se de uma pesquisa de abordagem qualitativa do tipo revisão integrativa da literatura, no ano 2020. Neste estudo foi inserido seis etapas a serem realizadas para adequado andamento do processo, sendo: estabelecimento do problema de revisão integrativa; estabelecido de critérios de inclusão/ exclusão de artigos (seleção da amostra); definição das informações a serem extraídas dos artigos selecionados; análise dos resultados; discussão e apresentação dos resultados; apresentação da revisão (Estrela, 2018).

\section{Procedimento para seleção dos artigos}

Utilizou-se a estratégia PICo, (P de "população/problema”, I de "Fenômeno de Interesse", Co de “contexto", acrônimo da língua inglesa primordial no delineamento do estudo e definidor das demais etapas da metodologia e processos seguintes do trabalho (Sousa et al., 2018; Santos, Pimenta \& Nobre, 2007). 
A questão de pesquisa foi estipulada como, qual o impacto da utilização de Cartas Terapêuticas (I) na comunicação (Co) entre equipe de saúde e pacientes $(\mathbf{P})$ ?

Quadro 1 - Apresentação do acrônimo estruturador da questão de pesquisa.

\begin{tabular}{|l|l|}
\hline P (População) & $\begin{array}{l}\text { Equipe de enfermagem e } \\
\text { pacientes }\end{array}$ \\
\hline I (Fenômeno de Interesse) & $\begin{array}{l}\text { Impacto do uso de cartas } \\
\text { terapêuticas }\end{array}$ \\
\hline Co (Contexto) & Comunicação \\
\hline
\end{tabular}

Fonte: pelos autores (2020).

A partir dessa fase metodológica foi possível definir como seria realizado o processo de obtenção dos dados nas bases de dados disponíveis. A revisão foi realizada nos meses de dezembro de 2020 e janeiro de 2021, por cinco revisores, via portal periódico Capes e foram selecionadas as bases de dados Cinahl, Medline e Scopus.

Quadro 2 - Os termos controlados utilizados foram selecionados dos tesauros Cinahl e DeCS/ MeSH, bem como os termos não controlados como apresentado a seguir:

\begin{tabular}{|l|l|}
\hline Bases de dados & Estratégia/Equação de busca \\
\hline CINAHL & therapeutic letters OR cartas terapêuticas AND health OR saúde \\
\hline MEDLINE & therapeutic letters OR cartas terapêuticas AND health OR saúde \\
\hline SCOPUS & therapeutic letters OR cartas terapêuticas AND health OR saúde \\
\hline
\end{tabular}

Fonte: Autores (2020).

Foram utilizados os seguintes critérios de inclusão (filtros): idioma português, inglês e espanhol, sem recorte temporal devido à escassez de materiais publicados, estudos em que os títulos e resumos contemplassem o assunto abordado. Os critérios de exclusão foram: artigos repetidos e artigos que tangenciassem o tema proposto pelo presente trabalho.

Essa revisão contou com cinco revisores e quando não houve consenso sobre inclusão/exclusão de registros, após a discussão optou-se pelo desempate. Primeiramente as obras foram armazenadas em planilha do programa Excel ${ }^{\mathbf{a}}$, em seguida foi realizada a pré-seleção de acordo com a leitura dos títulos e resumos. Nesta fase, buscou-se a relação entre o conteúdo do título e do resumo e se atendiam ao objetivo do presente estudo. Com isso, 24 artigos foram achados após a busca realizada nas bases. Após a leitura criteriosa de todo material detectou-se que um artigo estava duplicado e 13 artigos não atendiam ao tema proposto. Ao final, chegou-se a um compilado de 9 artigos como amostra final.

Para a avaliação dos artigos selecionados, visou-se os níveis de evidência que segundo Souza et al., (2010), seguem a hierarquização das classificações das metodologias que são dispostos como: nível 1 - evidências resultantes da meta-análise de múltiplos estudos clínicos controlados e randomizados; nível 2 - evidências obtidas em estudos individuais com delineamento experimental; nível 3 - evidências de estudos quase-experimentais; nível 4 - evidências de estudos descritivos (nãoexperimentais) ou com abordagem qualitativa; nível 5 - evidências provenientes de relatos de caso ou de experiência; nível 6 evidências baseadas em opiniões de especialistas. 


\section{Extração dos dados dos artigos incluídos na revisão integrativa}

Para a seleção das informações dos estudos, foi seguido a identificação dos seguintes itens: nome do autor; título do estudo; ano de publicação; nome do periódico; amostra do estudo; avaliação do rigor metodológico; objetivos e impacto na saúde da clientela.

\section{Resultados e Discussão}

A amostra final pode ser vista na imagem abaixo:

Figura 1 - Fluxograma da seleção de artigos

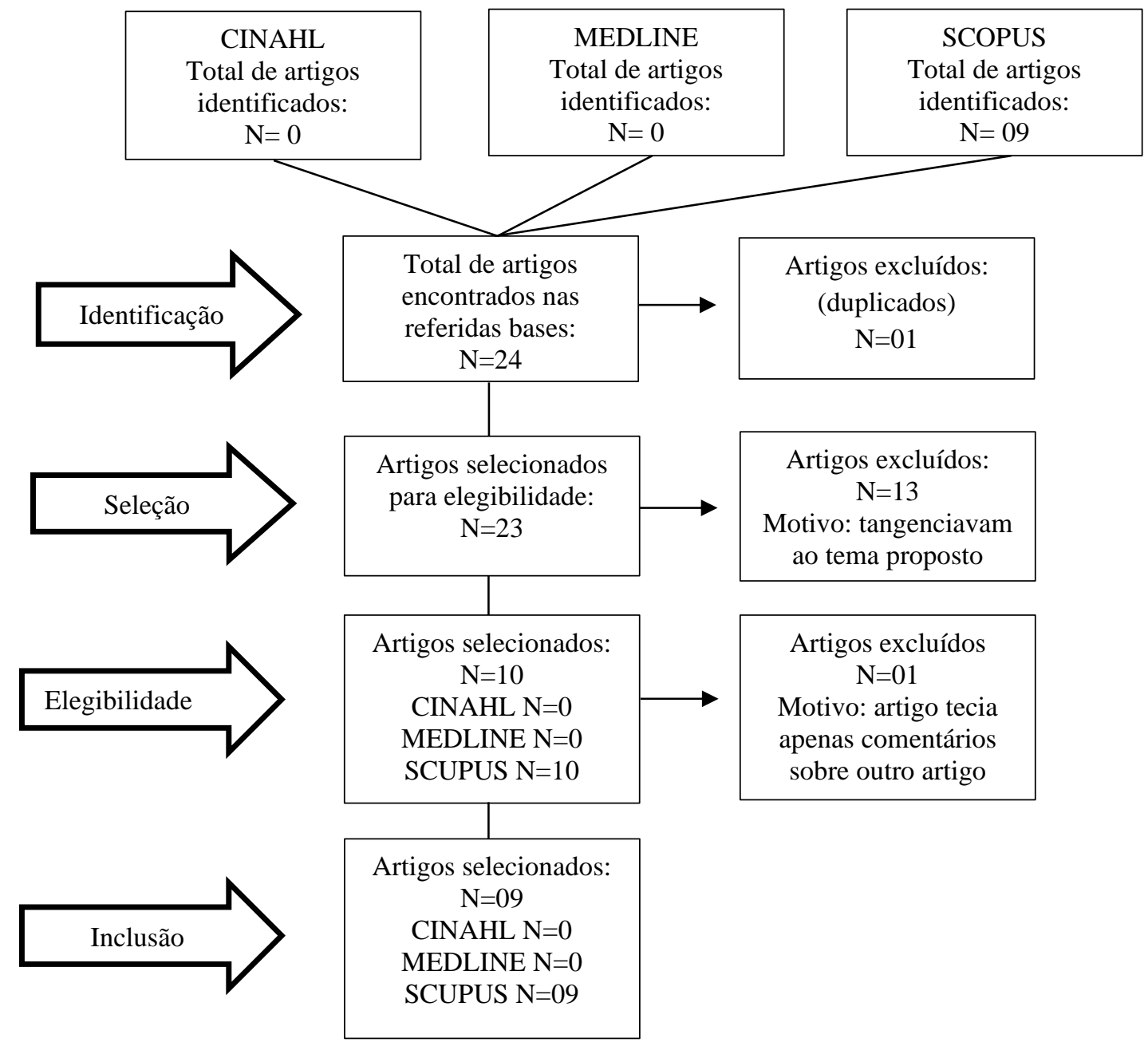

Fonte: Autores (2020).

A descrição geral, apresentada no quadro 3, detalha pontos específicos dos artigos encontrados a fim de chegar-se a um compilado importante e contributivo de cunho atualizado e fundamentado para a prática clínica, assim como preza a revisão integrativa. 
Research, Society and Development, v. 10, n. 8, e60010817687, 2021

(CC BY 4.0) | ISSN 2525-3409 | DOI: http://dx.doi.org/10.33448/rsd-v10i8.17687

Quadro 3 - Apresentação da síntese dos artigos selecionados na revisão integrativa.

\begin{tabular}{|c|c|c|c|c|c|}
\hline AUTOR & TÍTULO & ANO & $\begin{array}{l}\text { NOME DO } \\
\text { PERIÓDICO }\end{array}$ & AMOSTRA & $\begin{array}{l}\text { IMPACTO NA SAÚDE } \\
\text { DA CLIENTELA }\end{array}$ \\
\hline $\begin{array}{l}\text { Bell, Moules e } \\
\text { Wright }\end{array}$ & $\begin{array}{l}\text { Therapeutic Letters and } \\
\text { the Family Nursing Unit: } \\
\text { A Legacy of Advanced } \\
\text { Nursing Practice }\end{array}$ & 2009 & $\begin{array}{l}\text { Journal of Family } \\
\text { Nursing }\end{array}$ & $\begin{array}{l}\text { Cartas da } \\
\text { enfermagem para as } \\
\text { famílias. }\end{array}$ & $\begin{array}{l}\text { O artigo oferece exemplos de } \\
\text { uma variedade de cartas } \\
\text { terapêuticas escritas para } \\
\text { famílias que vivenciam o } \\
\text { sofrimento da doença. }\end{array}$ \\
\hline $\begin{array}{l}\text { Erlingsson, } \\
\text { Christen Lee. }\end{array}$ & $\begin{array}{l}\text { Undergraduate Nursing } \\
\text { Students } \quad \text { Writing } \\
\text { Therapeutic Letters to } \\
\text { Families: An Educational } \\
\text { Strategy }\end{array}$ & 2009 & $\begin{array}{l}\text { Journal of Family } \\
\text { Nursing }\end{array}$ & $\begin{array}{l}\text { Estudantes } \\
\text { enfermagem }\end{array}$ & $\begin{array}{l}\text { Os resultados indicam que } \\
\text { refletir sobre suas próprias } \\
\text { ações e sobre como a família } \\
\text { reagiria ajudou os alunos a } \\
\text { refletir-sobre-ação não } \\
\text { apenas retrospectivamente, } \\
\text { como na reflexão pós-ação, } \\
\text { mas também } \\
\text { prospectivamente. }\end{array}$ \\
\hline Pyle & $\begin{array}{ll}\text { Therapeutic } & \text { Letters as } \\
\text { Relationally } & \text { Responsive } \\
\text { Practice } & \end{array}$ & 2009 & $\begin{array}{l}\text { Journal of Family } \\
\text { Nursing }\end{array}$ & $\begin{array}{l}\text { Cartas de nove } \\
\text { médicos para } \\
\text { clientes adultos. }\end{array}$ & $\begin{array}{l}\text { Este artigo apresenta } \\
\text { resultados selecionados de } \\
\text { um estudo maior que } \\
\text { examinou as práticas de } \\
\text { escrever cartas de nove } \\
\text { médicos, bem como as } \\
\text { experiências de sete clientes } \\
\text { adultos que receberam uma } \\
\text { ou mais cartas terapêuticas } \\
\text { durante o curso de terapia } \\
\text { individual ou familiar. }\end{array}$ \\
\hline Neil Rodgers. & $\begin{array}{ll}\text { Therapeutic Letters: A } \\
\text { Challenge } & \text { to } \\
\text { Conventional Notions of } \\
\text { Boundary }\end{array}$ & 2009 & $\begin{array}{l}\text { Journal of Family } \\
\text { Nursing }\end{array}$ & $\begin{array}{l}\text { A experiência } \\
\text { De cinco dos clients } \\
\text { do autor que } \\
\text { concordaram em ser } \\
\text { entrevistados. }\end{array}$ & $\begin{array}{l}\text { A transposição dos limites } \\
\text { espaciais e temporais da } \\
\text { relação terapêutica. } \\
\text { Transcorrendo barreiras entre } \\
\text { o profissional e o pessoal na } \\
\text { relação entre o autor e a } \\
\text { clientela. }\end{array}$ \\
\hline $\begin{array}{l}\text { Mrduljaš Đujić et } \\
\text { al. }\end{array}$ & $\begin{array}{l}\text { Writing letters to patients } \\
\text { as an educational tool for } \\
\text { medical students. } \\
\text { (Report) }\end{array}$ & 2013 & $\begin{array}{l}\text { BMC Medical } \\
\text { Education }\end{array}$ & $\begin{array}{lr}108 & \text { cartas } \\
\text { terapêuticas de } \\
\text { pacientes escritas } \\
\text { por alunos como } \\
\text { uma ferramenta do } \\
\text { Ensino da medicina } \\
\text { familiar }\end{array}$ & $\begin{array}{l}\text { Valorização pela clientela } \\
\text { sobre a nova maneira de } \\
\text { aprendizado sobre sua } \\
\text { doença e formas de novos } \\
\text { estilos de vida. }\end{array}$ \\
\hline Moules, Nancy J & $\begin{array}{l}\text { Nursing on paper: } \\
\text { Therapeutic letters in } \\
\text { nursing practice }\end{array}$ & 2002 & Nursing Inquiry & $\begin{array}{l}4 \text { membros de uma } \\
\text { família e } 3 \\
\text { enfermeiros }\end{array}$ & $\begin{array}{l}\text { A dinâmica gerou satisfação } \\
\text { ao paciente em ter sua } \\
\text { necessidade reconhecida e } \\
\text { valorizada e seu sofrimento } \\
\text { aliviado. Sentiam-se ouvidos, } \\
\text { que os profissionais se } \\
\text { colocavam em seu lugar. }\end{array}$ \\
\hline Nancy J. Moules. & $\begin{array}{l}\text { Therapeutic Letters in } \\
\text { Nursing: Examining the } \\
\text { Character and Influence } \\
\text { of the Written Word in } \\
\text { Clinical Work With } \\
\text { Families Experiencing } \\
\text { Illness }\end{array}$ & 2009 & $\begin{array}{l}\text { Journal of Family } \\
\text { Nursing }\end{array}$ & $\begin{array}{l}11 \quad \text { cartas } \\
\text { terapêuticas escritas } \\
\text { por enfermeiras à } \\
\text { famílias no processo } \\
\text { de doença. }\end{array}$ & 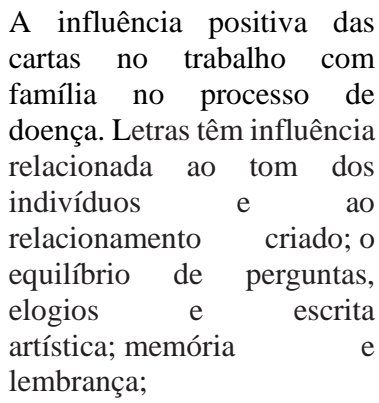 \\
\hline
\end{tabular}


Research, Society and Development, v. 10, n. 8, e60010817687, 2021

(CC BY 4.0) | ISSN 2525-3409 | DOI: http://dx.doi.org/10.33448/rsd-v10i8.17687

\begin{tabular}{|c|c|c|c|c|c|}
\hline $\begin{array}{l}\text { Freed, Patricia E.; } \\
\text { McLaughlin, } \\
\text { Dorcas E.; } \\
\text { Smithbattle, Lee; } \\
\text { Leander, Sheila; } \\
\text { Westhus, Nina }\end{array}$ & $\begin{array}{l}\text { Therapeutic letters in } \\
\text { undergraduate nursing } \\
\text { education: Ideas for } \\
\text { clinical nurse educators }\end{array}$ & 2010 & $\begin{array}{l}\text { Nurse Education } \\
\text { Today }\end{array}$ & $\begin{array}{l}\text { Escrita de cartas } \\
\text { terapêuticas }\end{array}$ & $\begin{array}{l}\text { Este artigo adiciona às } \\
\text { evidências que sustentam as } \\
\text { cartas terapêuticas como uma } \\
\text { estratégia eficaz para } \\
\text { promover as habilidades } \\
\text { relacionais dos alunos e } \\
\text { reconhecer os pontos fortes } \\
\text { dos pacientes. As cartas } \\
\text { terapêuticas têm benefícios } \\
\text { para alunos, pacientes e } \\
\text { professores. }\end{array}$ \\
\hline Smithbattle et al. & $\begin{array}{l}\text { Writing Therapeutic } \\
\text { Letters in Undergraduate } \\
\text { Nursing Education: } \\
\text { Promoting Relational } \\
\text { Skill Development }\end{array}$ & 2009 & $\begin{array}{l}\text { Qualitative Health } \\
\text { Research }\end{array}$ & $\begin{array}{l}\text { Aprendizagem } \\
\text { clínica de estudantes } \\
\text { na graduação de } \\
\text { enfermagem }\end{array}$ & $\begin{array}{l}\text { As cartas terapêuticas } \\
\text { cultivam a construção e o } \\
\text { desenvolvimento das } \\
\text { habilidades relacionais dos } \\
\text { alunos. Embora a tarefa tenha } \\
\text { promovido o aprendizado } \\
\text { clínico e a reflexão sobre as } \\
\text { relações. }\end{array}$ \\
\hline
\end{tabular}

Fonte: Autores (2021).

O desenvolvimento interpessoal entre paciente e o profissional construído através das cartas terapêuticas dos estudos envolvidos no compilado filtrado nas bases de dados já descritas no presente trabalho se apresentou de maneira muito semelhante nos 9 variados artigos selecionados a partir da busca. A construção de uma base sólida no processo de intervenção do cuidado em clientes e familiares acometidos pelo processo de doença foi imprescindível, assim como mostram os estudos.

Segundo Moules (2009) os ganhos não são somente para quem recebe as cartas terapêuticas, ou seja, o remetente, mas também, para aqueles que se esforçam para a construção de terapias em forma de palavras, já que esse movimento exige do emissor o desenvolvimento de habilidades profissionais de comunicação. Por esse motivo, muitos artigos abordam o tema das cartas terapêuticas de forma correlata à implantação dessa nova maneira de transpassar os limites dos cuidados tradicionais dentro de cursos de especialização profissional.

De acordo com Dujic (2013) a tecnologia a serviço da educação se encontra nesse desabrochar da inovação, o que gera um novo olhar, possibilita dar a mesma prioridade ao aprendizado de habilidades relacionais como é dado ao desempenho de habilidades técnicas. Essa máxima é uma crescente e precisa ser destacada cada vez mais, assim ganhando espaço no mundo científico e acadêmico, de forma suficientemente necessária a se tornar parte das grades curriculares de formação. Construindo uma educação profissional em saúde forte baseada no raciocínio ético, no raciocínio e julgamento clínico e nas necessidades, anseios e preocupações do paciente.

Para Rodgers (2009) é poder ultrapassar os limites impostos por uma forma de promoção de saúde ensinada há décadas. É estabelecer pilares e instituir meios facilitadores de sempre estar ao lado do paciente, e que pode ser lidas repetidas vezes, a carta terapêutica vem com essa proposta para implantação, uma tecnologia de comunicação atemporal. Facilitadora da referência e contrarreferência do cuidado integral de qualidade e do alcance de resultados clínicos positivos.

Segundo Bell, Moules \& Wright (2009) as cartas terapêuticas proporcionam uma melhor comunicação entre profissional da saúde e paciente, dando ênfase na informação necessária, pontuando situações, permitindo apaziguar o paciente proporcionando oportunidade de explicação e soluções de problemas de uma forma estratégica, diminuindo assim, a defensiva dos profissionais e facilitando o entendimento dos pacientes, consequentemente melhorando a adesão e compreensão do tratamento. Também proporcionam oportunidades de encorajar, elogiar e reconhecer a força individual e dos familiares. 
Pyle (2009) diz que as cartas terapêuticas "tocam" no destinatário em um tom mais leve, com a voz do "outro" (remetente), levando mais engajamento e diálogo em torno do processo terapêutico, retirando os profissionais responsáveis do lugar de espectadores ou passivos participantes e levando-os para uma posição de mais responsabilidade e atividade com o tratamento.

Erlingsson (2009), evidencia em seu trabalho a necessidade de incentivo que a maioria dos alunos que escreveram cartas terapêuticas apresentou para renunciar ao papel de detentores do saber, à posição hierárquica de enfermeiros especialistas e focar nos pontos fortes e recursos da família em questão. Pode-se inferir, ainda, a importância da reflexão acerca das conexões entre a teoria apreendida nos módulos de enfermagem familiar e a própria realidade das famílias.

O estudo promovido por Smithbattle et al., (2009) diz que as cartas terapêuticas tornaram-se um meio de comunicação eficaz entre pacientes e profissionais, de experiência importante para a formação profissional dos remetentes, onde a escrita proporcionou criar habilidades e a reflexão sobre o desenvolvimento de ajudar relações. Também foram identificados benefícios para os pacientes em que as cartas foram destinadas, criou-se a oportunidade de validar seus aspectos de vida e reforçar os seus objetivos, conquistas e novas práticas de saúde.

A escrita de cartas terapêuticas requer respeito e atenção, bem como o reconhecimento de que as palavras escritas serão lidas por pessoas com seus espíritos e corpos, exercerão influência em suas vidas, relacionamentos e deve-se lembrar, ainda, que podem curar ou machucar. Há que se ter ética e responsabilidade ao escrever cartas terapêuticas para fazê-lo bem (MOULES, 2002).

\section{Contribuições dos autores para a prática clínica de enfermagem}

Uma carta terapêutica escrita de modo centrado na pessoa contribui para a assistência dada ao paciente. São escritas em um estilo que transmite compaixão, valida os pontos fortes, reconhece realizações, força e caráter e aprofunda relações de confiança. Escrever uma carta terapêutica para um paciente é uma intervenção usada por muitos terapeutas, enfermeiros e conselheiros para melhorar ou complementar o processo terapêutico (FREED et al., 2010).

A carta deverá ser apresentada em uma folha A4, em único verso de folha, porque possibilita a comunicação direta entre profissional e paciente, de acordo com uma interface objetiva, escrita a caneta pelo próprio punho de forma a aproximar as relações interpessoais e compor suporte emocional, diante das mazelas do cotidiano vivenciado no período da pandemia, por profissionais de saúde que se mostraram dispostos a realizar tal ação a partir de um diagnóstico situacional comum a um olhar científico da prática da equipe de enfermagem que possibilita o planejamento e a implementação de uma estrutura mais sólida no elo paciente-profissional, compondo uma assistência mais humanizada, integral e universal.

Conteúdo da Carta: "Cara (o) (nome do paciente), lhe escrevo esta pequena cartinha para dizer que sempre estarei com você! Que sua luta é um pouco da minha luta de todos os dias. Quando conhecemos alguém, deixamos um pouco de nós e levamos muito dos outros. Diariamente estamos com você, enfrentando dias positivos e aqueles que não são tão bons e positivos assim, não é isso?!

Construímos laços e o fortalecemos mais a cada instante e a cada cuidado. O cuidado é a nossa ciência e arte, no qual nós dedicamos anos de estudos para construirmos uma assistência de qualidade que atenda a todas as demandas vindas de você. Somos profissionais do cuidado e não deixamos de ser seres humanos, assim como você sentimos medo, principalmente neste cenário de pandemia em que a possibilidade de adoecermos e aos nossos entes queridos é grande, sentimos dor, frio, amor, carinho, saudades do contato com amigos e familiares e é por isso que escrevemos. Escrevemos porque sentimos a necessidade de deixar registrado o nosso carinho e cuidado por você. 
Você é IMPORTANTE para nós. A sua saúde e a sua recuperação é o que nós mais queremos e rezamos! Estamos com você em mais essa batalha, nem sempre seremos as mesmas pessoas, nos mesmos locais para atendê-la, mas esteja certa que estamos preparados para minimizar as feridas dessa batalha sempre que for necessário.

Assinado, equipe de enfermagem."

A dinâmica da distribuição da carta: a distribuição das cartas se dará por meio da equipe de enfermagem durante a tarde, devido ao grande número de práticas de assistência e cuidados de enfermagem durante a rotina matutina das enfermarias. Assim, serão entregues para todos os pacientes internados na unidade. Essas cartas irão conter o mesmo conteúdo, de formato padrão de forma individualizada, mas focalizado no contexto atual vivenciados por todos, tanto profissionais, quanto pacientes. Nelas haverá peculiaridades envolvidas, como o nome/sobrenome/nome social, respeitando sua preferência quanto a identificação do paciente a qual era entregue, de forma que isso possa iniciar uma reflexão, já bem no início, a partir da sua própria identificação na escrita e através do seu reconhecimento e inclusão naquilo que estava lendo. Entendendo, dessa maneira, que ele importava e se importavam com sua estada naquele meio e durante a situação vivida. Os pacientes que não soubessem ler ou com dificuldade de leitura, um dos membros da equipe grava um vídeo lendo esta carta e manda, via telefone celular, através de aplicativo de conversas.

\section{Considerações Finais}

A construção do presente estudo buscou enfatizar a necessidade de um olhar diferenciado e minucioso para os desdobramentos patológicos na saúde mental dos pacientes, através da busca de material científico que mostrasse e comprovasse a importância, no processo de saúde-doença, do tratamento psicológico, de acordo com medidas efetivas no alívio de tensões e ansiedades geradas pelos efeitos do adoecimento humano. Além disso, trazer novas reflexões acerca do papel do profissional enfermeiro na tentativa, através de manejo científico e embasado, de redução de danos causados a partir dos impactos trazidos pelo coronavírus às novas realidades.

Acredita-se que a representação psíquica que a carta terapêutica ocupou para o paciente, e o significado simbólico das palavras da carta, pode ter contribuído de alguma forma na busca de uma ressignificação daquele momento de isolamento social. Arrisca- se ainda a acreditar que o recebimento desta carta desperta sentimentos que permite incentivar a construção de processos psíquicos, que podem ser favoráveis à cura ou ao completo bem-estar biopsicossocial.

É importante ressaltar o teor iminente da temática estudada e as implicações associadas a esse caráter. É escassa a produção científica sobre o assunto supracitado e, portanto, diminuído está o embasamento científico para esta obra, por esse motivo é necessário o incentivo a esses assuntos referenciados ao cunho holístico do ser humano.

Nessa perspectiva, é inovadora e diferencial a aplicabilidade das cartas terapêuticas para relações interpessoais, o que pode evidenciar um cuidado mais humanizado por parte da equipe de enfermagem, motivando o paciente a entender que a equipe está junto a ele em todo o processo de recuperação e tratamento, estabelecendo um vínculo de confiança, referência e contrarreferência na assistência. Ratificando um dos grandes princípios do Sistema Único de Saúde (SUS), a integralidade da assistência, através de uma visão holística, prestando suporte emocional e físico necessário a cada indivíduo como ser social único.

Para trabalhos futuros, seria interessante um aprofundamento em relação aos efeitos gerados ao cliente no processo terapêutico após receber a carta e uma ampla divulgação destes para toda a equipe, além da investigação de possíveis mudanças durante essa prática, deixando explícito na literatura todos seus benefícios e impulsionando a adesão dessa estratégia na rotina 
de trabalho.

\section{Referências}

Bell, J. M., Moules, N. J. \& Wright, L. M. (2009). Therapeutic Letters and the Family Nursing Unit. Journal Of Family Nursing, (15)1: 6-30. https://journalssagepub-com.ez39.periodicos.capes.gov.br/doi/pdf/10.1177/1074840709331865.

Erlingsson, C. (2009). Undergraduate Nursing Students Writing Therapeutic Letters to Families. Journal Of Family Nursing, (15)1: 83-101.

Estrela, C. (2018). Metodologia Científica: Ciência, Ensino, Pesquisa. Editora Artes Médicas.

Fernandes, C. et al. (2015). The Importance of Families in Nursing Care: nurses: attitudes in the hospital environment. Revista de Enfermagem Referência. 7: 21-30.

Freud, S. (1969). Estudos sobre a Histeria. Edição Standard Brasileira das Obras Psicológicas Completas de Sigmund Freud. Volume II. Rio de Janeiro.

Freud, S (1969). A dinâmica da Transferência. Edição Standard Brasileira das Obras Psicológicas Completas de Sigmund Freud. Volume XII. Rio de Janeiro.

Hunt, C., Shochet, I. \& King, R. (2005). The Use of E-mail in the Therapy Process. Australian And New Zealand Journal Of Family Therapy, (26)1:10-20. http://dx.doi.org/10.1002/j.1467-8438.2005.tb00631.x.

Freed, P. E., McLaughlin, D. E., Smithbattle, L., Leander, S. \& Westhus, N. (2010). Therapeutic letters in undergraduate nursing education: ideas for clinical nurse educators. Nurse Education Today, (30)5: 470-475. http://dx.doi.org/10.1016/j.nedt.2009.10.009.

Maio, A. M. D. (2020). Abraços digitais, cartas e crachás humanizados. Anais de Resumos Expandidos do Seminário Internacional de Pesquisas em Midiatização e Processos Sociais, (1) 4. https://midiaticom.org/anais/index.php/seminario-midiatizacao-resumos/article/view/1150.

Moules, N. J. (2002). Nursing on paper: therapeutic letters in nursing practice. Nursing Inquiry, (9)2: 104-113. http://dx.doi.org/10.1046/j.14401800.2002.00133.x

Moules, N. J. (2009). Therapeutic Letters in Nursing Examining the Character and Influence of the Written Word in Clinical Work With Families Experiencing Illness. Journal of Family Nursing, (15)1:31-49.

Dujic, N. M., Zitnic, E., Pavelin, L., Bacic, D., Boljat, M., Vrdoljak, D., Pavlicevic, I., Dvornik, A. \& Marusic, M. (2013). Writing letters to patients as an educational tool for medical students. Gale academic onefile. BMC Medical Education. BioMed Central Ltd. v.13, n.1.

Oliver, M., Nelson, K. W., Cade, R. \& Cueva, C. (2007). Therapeutic Letter Writing from School Counselors to Students, Parents, and Teachers. Professional School Counseling. (10)5: 510-515. https://www.jstor.org/stable/42732727?seq=1.

Ornell, F., Schuch, J. B., Sordi, A. O. \& Kessler, F. H. P. (2020). "Pandemic fear" and COVID-19: Mental health burden and strategies. Braz J Psychiatry. 42: 232-235.

Paiva, L. P. C. \& Rasera, E. F. (2012). O uso das cartas terapêuticas na prática clínica. Psicologia Clínica. (24)1: 193-207. https://www.scielo.br/pdf/pc/v24n1/13.pdf.

Pyle, N. R. (2009). Therapeutic Letters as Relationally Responsive Practice. Journal Of Family Nursing. (15)1: 65-82. https://journals-sagepubcom.ez39.periodicos.capes.gov.br/doi/pdf/10.1177/1074840709331640.

Raony, Í., Figueiredo, C. S., Pandolfo, P., Araujo, E. G., Bomfim, P. O. S. \& Savino, W. (2020). Psycho-Neuroendocrine-Immune Interactions in COVID-19: potential impacts on mental health. Frontiers In Immunology. (11):1-15. https://www.frontiersin.org/articles/10.3389/fimmu.2020.01170/full.

Rodgers, N. (2009). Therapeutic letter a challenge to conventional notions of boundary. Journal of Family Nursing. (15)1: 50-64.

Santos, C. M. D. C., Pimenta, C. A. D. M., \& Nobre, M. R. C. (2007). A estratégia PICO para a construção da pergunta de pesquisa e busca de evidências. Revista Latino-Americana de Enfermagem, 15, 508-511.

Schmidt, B., Crepaldi, M. A., Bolze, S. D. A., Silva, L. N. \& Demenech, L. M. (2020). Saúde mental e intervenções psicológicas diante da pandemia do novo coronavírus (COVID-19). Estudos de Psicologia (campinas). (37)1: 1-13. https://www.scielo.br/scielo.php?script=sci_arttext\&pid=S0103166 X2020000100501\&tlng=pt.

Smithbattle, L., Leander, S., Westhus, N., Freed, P. E. \& McLaughlin, D. E. (2009). Writing Therapeutic Letters in Undergraduate Nursing Education: promoting relational skill development. Qualitative Health Research. (20)5: 707-716.

Sousa, L. M. M., Marques, J. M., Firmino, C. F., Frade, F., Valentim, O. S. \& Antunes, A. V. (2018). Modelos de formulação da questão de investigação na Prática Baseada na Evidência. Revista Investigação Enfermagem. (23): 31-39. https://repositorio-cientifico.essatla.pt/bitstream/20.500.12253/1287/1/artigo3139.pdf.

Souza, M. T.; Silva, M. D. \& Carvalho, R. (2010). Revisão integrativa: o que é e como fazer, einstein; 8 (1 Pt 1$): 102-6$. https://www.scielo.br/pdf/eins/v8n1/pt_1679-4508-eins-8-1-0102.

Vidgen, A. \& Williams, R. (2001). Letter-writing practices in a child and family service. Journal Of Family Therapy. (23)3: 317-326. https://onlinelibrary.wiley.com/doi/epdf/10.1111/1467-6427.00186. 